\title{
A FUNCTORIAL APPROACH TO THE ONE-VARIABLE JONES POLYNOMIAL
}

\author{
R. J. LAWRENCE
}

\begin{abstract}
In this paper, a representation of the category of tangles will be given, which has a natural meaning in terms of certain twisted homology groups. The representation is demonstrated explicitly in terms of a presentation of this category found by Turaev. The invariant of links obtained is identified with the one-variable Jones polynomial via the skein relation, and some remarks are made on how the procedure can be extended to give the two-variable Jones polynomial.
\end{abstract}

\section{Introduction}

In [10] it was shown how an operator invariant of tangles could be obtained for any enhanced Yang-Baxter operator. The techniques of quantum groups (introduced by Drinfel'd [1], Jimbo [2] and others) may be used to produce a solution of the (constant quantum) Yang-Baxter equation,

$$
R_{12} R_{13} R_{23}=R_{23} R_{13} R_{12},
$$

where $R \in \operatorname{End} V^{\otimes 2}$, and $R_{i j} \in$ End $V^{\otimes 3}$ denotes the action of $R$ on the $i$ th and $j$ th factors, and the identity on the third factor. This may be extended to give an enhanced Yang-Baxter operator for any Lie group and associated representation. In this paper, we will consider the simplest case of $\mathfrak{s l}_{2}$ and the vector (spin-1/2) representation, constructing the representation of the category of tangles using topology. The images of objects in the category of tangles will be homology groups, evaluated on a configuration space of points in a punctured complex plane, using a twisted local coefficient system based on $\mathbb{C}^{*}$. Such homology groups were studied in detail in [5] and it was seen there how representations of Hecke algebras could be obtained from them (see $\S 2$ for a summary).

The construction of the representation of the category of tangles is carried out in detail in $\S 3$, where explicit images of the generators are given,

Received May 14, 1991 and, in revised form, February 18, 1992. This work was supported in part by National Science Foundation Grant DMS-9013738. This paper was written while the author was a Lindemann Fellow of the English Speaking Union. She is now a Junior Fellow of the Society of Fellows at Harvard University. 
using Turaev's presentation of the category [11]. The invariant of links so obtained is easily seen to satisfy a skein relation, and can thereby be identified with the (one-variable) Jones polynomial, $V$. In [3], the $V$ polynomial was expressed, in terms of a braid group description of the link, as a combination of characters of the Iwahori-Hecke algebra. The relation between the braid group and link diagram approaches to $V$ is discussed in $\S 4$, via an algebraic result on Hecke algebra representations. Another approach to links via braids, using the plait closure rather than braid closure, is investigated in $\S 5$, and the paper concludes with some remarks on extensions of the approach for the two-variable Jones polynomial $P($ or $X)$.

\section{Review of standard theory}

2.1. Braid group representations. In this section we shall review the topological constructions of [5]. Let $X_{n}\left(\widetilde{X}_{n}\right)$ be the configuration space of $n$ distinct ordered (unordered) points in the complex plane. Then there is a natural fibration of $X_{m+n}$ over $X_{n}$ with fibre over the point $\mathbf{w}=\left(w_{1}, \ldots, w_{n}\right) \in X_{n}$, given by the configuration space of $m$ distinct points in the punctured complex plane $\mathbb{C} \backslash\left\{w_{1}, \ldots, w_{n}\right\}$. Let $Y_{\mathbf{w}, m}=$ $\left\{\left(z_{1}, \ldots, z_{m}\right) \in X_{m} \mid z_{i} \neq w_{j}, \forall 1 \leq i \leq m, 1 \leq j \leq n\right\}$ denote this fibre.

The fundamental groups $\pi_{1}\left(X_{n}\right)$ and $\pi_{1}\left(\tilde{X}_{n}\right)$ are the pure and full braid groups on $n$ strings, $P_{n}$ and $B_{n}$, respectively. Similarly $\pi_{1}\left(Y_{\mathrm{w}, m}\right)$ is a generalized version of $P_{m}$ in which $\mathbb{C}$ has been replaced by a punctured complex plane. For any representation (character),

$$
\chi: \pi_{1}\left(Y_{\mathbf{w}, m}\right) \rightarrow \mathbb{C}^{*}
$$

one can define a local coefficient system on $Y_{\mathbf{w}, m}$, based on $\mathbb{C}^{*}$. The group $\pi_{1}\left(Y_{\mathbf{w}, m}\right)$ is generated by $\left\{\beta_{\lambda, \mu}\right\}$ where $\lambda \in\left\{z_{1}, \ldots, z_{m}\right\}$ and $\mu \in\left\{z_{j+1}, \ldots, z_{m}, w_{1}, \ldots, w_{n}\right\} \quad\left(\lambda=z_{j}\right)$. The loop $\beta_{\lambda, \mu}$ is specified by a motion of $\mathbf{z}$ in which $z_{k}$ is fixed $\forall k \neq j$, and $z_{j}$ follows a loop around $\mu$ with winding number -1 , as shown in Figure 1. We here fix a base-point $\mathbf{z}^{0} \in Y_{\mathbf{w}, m}$, for which $\Im\left(z_{1}^{0}\right)<\cdots<\Im\left(z_{m}^{0}\right)<\Im\left(w_{1}\right)<\cdots<$ $\Im\left(w_{n}\right)$, and for the purpose of this part of the discussion, $\mathbf{w}$ is fixed. It is now possible to choose $\chi$ so that,

$$
\begin{aligned}
& \chi\left(\beta_{z_{j} z_{k}}\right)=q^{2} \quad \forall k>j, \\
& \chi\left(\beta_{z_{j} w_{i}}\right)=q^{-1} \quad \forall i, j
\end{aligned}
$$




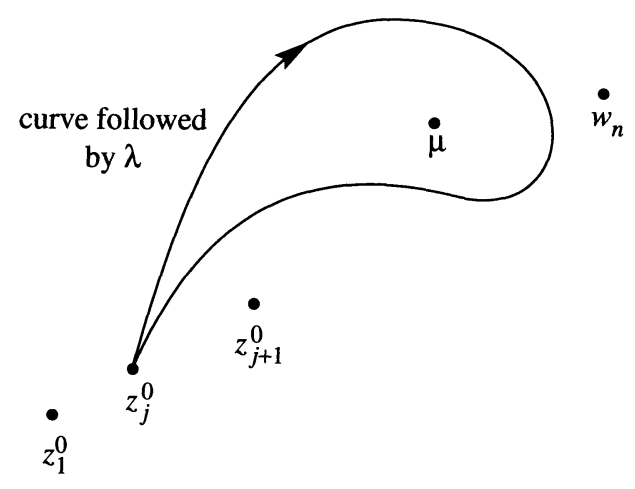

FIGURE 1

(the abelianisations of all relations on $\pi_{1}\left(Y_{w, m}\right)$ are trivial). Then $H^{m}\left(Y_{\mathrm{w}, m}, \chi\right)$ defines the fibre of a vector bundle $E_{m}(q)$ over the base space $X_{n}$. Since $\chi$ is invariant under the action of the subgroup $S_{m} \times S_{n}$ of $S_{m+n}$ which permutes either or both of the sets $\left\{w_{i}\right\}$ and $\left\{z_{j}\right\}$, we obtain a vector bundle $\widetilde{E}_{m}(q)$, fibred over the base space $\widetilde{X}_{n}$, with fibre $H^{m}\left(Y_{\mathbf{w}, m} / S_{m}, \chi\right)$.

Theorem 2.1 [5]. The action of $B_{n}$ on $H^{m}\left(Y_{\mathrm{w}, m} / S_{m}, \chi\right)$ contains, as a subrepresentation, the action of the representation of $B_{n}$ which factors through the Hecke algebra $H_{n}(q)$ and is associated with the two-row Young diagram $\Lambda_{m}^{n}$.

In this theorem, the Hecke algebra $H_{n}(q)$ is a quadratic algebra quotient of the group algebra $\mathbb{C} B_{n}$ of the braid group $B_{n}$. In terms of generators and relations, $B_{n}$ has generators $\left\{\sigma_{1}, \ldots, \sigma_{n-1}\right\}$ and relations,

$$
\begin{aligned}
\sigma_{i} \sigma_{j} & =\sigma_{j} \sigma_{i}, & & |i-j|>1, \\
\sigma_{i} \sigma_{i+1} \sigma_{i} & =\sigma_{i+1} \sigma_{i} \sigma_{i+1}, & & i=1,2, \ldots, n-2 .
\end{aligned}
$$

The algebra $H_{n}(q)$ is generated by $\left\{1, \sigma_{1}, \ldots, \sigma_{n-1}\right\}$ as an algebra, with, in addition to the two above relations, the extra relation,

$$
\left(\sigma_{i}-1\right)\left(\sigma_{i}+q\right)=0, \quad i=1,2, \ldots, n-1 .
$$

This defines the $A_{n-1}^{(1)}$-Hecke algebra; it is a deformation of $\mathbb{C} S_{n}$ when $q$ moves away from 1 . The representation theory of $H_{n}(q)$ is similar to that of $S_{n}$, when $q$ is not a root of unity (see [12]); that is, for any Young diagram, $\Lambda$, with $n$ squares, there is defined a representation $\pi_{\Lambda}$ of $H_{n}(q)$ which deforms the standard representation of $S_{n}$ associated with $\Lambda$, as $q$ moves away from 1 . The particular representation referred 
to in the statement of the theorem is that connected with the two-row Young diagram $\Lambda_{m}^{n}$ in which the rows have lengths $n-m$ and $m$.

Let $\mathscr{S}_{\mathbf{w}}^{m}$ denote the set of all $\underline{\alpha}=\left(\alpha_{1}, \ldots, \alpha_{m}\right)$ where $\alpha_{i} \in\left\{z_{i+1}, \ldots\right.$, $\left.z_{m}, w_{1}, \ldots, w_{n}\right\}$. Here $z_{1}, \ldots, z_{m}$ and $w_{1}, \ldots, w_{n}$ are to be treated as formal symbols. Let $\mathscr{U}_{\mathrm{w}}^{m}$ and $\mathscr{T}_{\mathrm{w}}^{m}$ denote the subsets of $\mathscr{S}_{\mathrm{w}}^{m}$ defined by

and

$$
\alpha_{1}>\alpha_{2}>\cdots>\alpha_{m}>z_{m}
$$

$$
\alpha_{i} \in\left\{w_{1}, \ldots, w_{n}\right\} \quad \forall i \text { with } \alpha_{i} \neq \alpha_{j} \forall i \neq j
$$

respectively. We have here defined an ordering on the symbols $z_{1}, \ldots, z_{m}$, $w_{1}, \ldots, w_{n}$ so that this sequence is in ascending order.

Fix $\mathbf{w} \in X_{n}$ with $\left\{\Im\left(w_{i}\right)\right\}_{i=1}^{n}$ increasing. Choose a base-point $\mathbf{z}^{0} \in Y_{\mathbf{w}, m}$ for which $\Im\left(z_{1}^{0}\right)<\cdots<\Im\left(z_{m}^{0}\right)<\Im\left(w_{1}\right)$. For any $j(1 \leq j \leq m)$ and $\mu \in\left\{z_{j+1}, \ldots, z_{m}, w_{1}, \ldots, w_{n}\right\}$, define a loop in $Y_{w, m}$, denoted $\beta_{z_{j} \mu}$, with base-point $\mathbf{z}^{0}$, for which $z_{i}(i \neq j)$ is constant at $z_{i}^{0}$ throughout, while $z_{j}$ follows a loop around the base-point value of $\mu$. This defines a unique element of $\pi^{1}\left(Y_{\mathbf{w}, m}\right)$ when it is stipulated that the path of $z_{j}$ does not cross any of the rays $\mathbb{R}^{+}+x$ for $x \in\left\{z_{1}^{0}, \ldots, z_{m}^{0}, w_{1}, \ldots, w_{n}\right\}$, $x \neq z_{j}^{0}, \mu$ while having winding number -1 around $\mu$.

For any $\underline{\alpha} \in \mathscr{S}_{\mathbf{w}}^{m}$, define an embedding,

$$
\gamma_{\underline{\alpha}}:[0,1]^{m} \rightarrow Y_{\mathbf{w}, m}
$$

with base-point $\mathbf{z}^{0}$, so that $\left(\gamma_{\underline{\alpha}}\left(t_{1}, \ldots, t_{m}\right)\right)_{i}$ is independent of $t_{1}, \ldots$, $t_{i+1}$. The $m$ th component is defined to be $\left(\beta_{z_{m} \alpha_{m}}\left(t_{m}\right)\right)_{m}$ while the $i$ th component is a function of $t_{i}, \ldots, t_{m}$ which is such that it is $\left(\beta_{z_{i} \alpha_{i}}\left(t_{i}\right)\right)_{i}$ at $t_{i+1}=\cdots=t_{m}=0$, and a continuous deformation of this loop when $\left(t_{i+1}, \ldots, t_{m}\right)$ moves away from the origin so moving $z_{i+1}, \ldots, z_{m}$ away from their base-point positions. This embedding defines $\left[\gamma_{\underline{\alpha}}\right] \stackrel{m}{\epsilon}$ $H_{m}\left(Y_{\mathrm{w}, m}, \chi_{0}\right)$ where $\chi_{0}$ is the trivial local coefficient system. Let $\widetilde{\widetilde{Y}}_{\mathrm{w}, m}$ denote the cyclic covering of $Y_{\mathbf{w}, m}$ defined by the map,

$$
\begin{aligned}
\pi_{1}\left(Y_{\mathrm{w}, m}\right) & \rightarrow \mathbb{Z}, \\
\beta_{z_{i} z_{j}} & \mapsto 2, \\
\beta_{z_{i} w_{k}} & \mapsto-1
\end{aligned}
$$

for all $1 \leq i, j \leq m, 1 \leq k \leq n$. The local coefficient system $\chi$ is thus defined in terms of $\widetilde{Y}_{w, m}$ as the local coefficient system which scales by 
$q$ under the natural translation on $\tilde{Y}_{\mathrm{w}, m}$, which shifts one branch of the cover up to the next. The embedding of the hypercube $[0,1]^{m}$ in $Y_{\mathbf{w}, m}$ specified by $\gamma_{\underline{\alpha}}$ lifts to an embedding of $[0,1]^{m}$ in $\tilde{Y}_{\mathbf{w}, m}$.

It can be shown that the homology $H_{m}\left(Y_{\mathrm{w}, m}, \chi\right)$ may be identified with the kernel of a boundary map $\delta$ on the vector space, $V$, with one basis element for each $\underline{\alpha} \in \mathscr{S}_{\mathrm{w}}^{m}$. Dually, one may identify $H^{m}\left(Y_{\mathrm{w}, m}, \chi\right)$ as a quotient space of a vector space of dimension $\left|\mathscr{S}_{\mathbf{w}}^{m}\right|$. Essentially, it can be thought of as a space of functions, $f$, on $Y_{\mathbf{w}, m}$ which twist according to $\chi$, up to an identification in which $\partial f / \partial z_{i} \sim 0 \forall f, i$. Let $(\underline{\alpha})$ denote the element of cohomology represented by that function $f$ for which,

$$
\int_{\gamma_{\underline{\beta}}} f=\delta_{\underline{\alpha} \underline{\beta}}
$$

$\forall \underline{\alpha}, \underline{\beta} \in \mathscr{S}_{\mathbf{w}}^{m}$. Then these elements span the cohomology, with various relations existing between them. It was shown in [5] that the representation of Theorem 2.1 is obtained on a specific subspace of the cohomology space, which we now proceed to construct.

Definition 2.2. $f_{\underline{\alpha}}=\sum_{\sigma \in S_{m}} q^{\varepsilon(\sigma)}(\sigma(\underline{\alpha}))$ for $\underline{\alpha} \in \mathscr{U}_{\mathrm{w}}^{m}$.

In this definition $\sigma(\underline{\alpha})$ denotes the image of $\underline{\alpha} \in \mathscr{U}_{\mathrm{w}}^{m}$ under the natural action of $\sigma \in S_{m}$, by permuting the $m$ components of $\underline{\alpha}$, as an element of $\mathscr{T}_{\mathrm{w}}^{m}$. Also $\varepsilon(\sigma)$ denotes the number of pairs $(i, j)$ with $1 \leq i<j \leq m$ for which $\sigma(i)>\sigma(j)$.

Lemma 2.3. $\left\{f_{\underline{\alpha}} \mid \underline{\alpha} \in \mathscr{U}_{\mathrm{w}}^{m}\right\}$ spans a subspace of $V$ and defines $a$ subspace of the cohomology space $H^{m}\left(Y_{\mathrm{w}, m}, \chi\right)$ on which the relations satisfied by the $f_{\underline{\alpha}}$ are,

$$
\sum_{w_{i} \notin \underline{\alpha}} q^{s_{i}} f_{\underline{\alpha} w_{i}}=\mathbf{0}
$$

for all $\underline{\alpha} \in \mathscr{U}_{\mathrm{w}}^{m-1}$ where $s_{i}$ is such that $\alpha_{s_{i}-1}>i>\alpha_{s_{i}}, 1 \leq s_{i} \leq m$. Here $f_{\underline{\alpha} w_{i}}$ refers to $f_{\underline{\beta}}$ where $\underline{\beta} \in \mathscr{U}_{\mathrm{w}}^{m}$ is the sequence $\underline{\alpha} w_{i}$ reordered so as to be in decreasing order.

The subspace of Lemma 2.3 is invariant under the action of $S_{m}$, and the action of $\sigma_{i} \in B_{n}$ upon it is given explicitly by,

$$
\sigma_{i}\left(f_{\underline{\alpha}}\right)= \begin{cases}f_{\underline{\alpha}_{i}}+(1-q) f_{\underline{\alpha}} & \text { if } w_{i} \in \underline{\alpha}, w_{i+1} \notin \underline{\alpha}, \\ q f_{\underline{\alpha}_{i}} & \text { if } w_{i} \notin \underline{\alpha}, w_{i+1} \in \underline{\alpha}, \\ f_{\underline{\alpha}} & \text { otherwise, }\end{cases}
$$


where $\underline{\alpha}_{i}$ denotes the sequence obtained from $\underline{\alpha} \in \mathscr{U}_{\mathrm{w}}^{m}$ by interchanging $w_{i}$ and $w_{i+1}$. It was also shown in [5] that the character of the representation of $H_{n}(q)$ on $\left\langle f_{\underline{\alpha}}\right\rangle$, specified by the action of $\sigma_{i}$ given above, is $\chi_{\Lambda_{1}^{n}}+\cdots+\chi_{\Lambda_{m}^{n}}$; while the relations of Lemma 2.3 transform according to the character $\chi_{\Lambda_{1}^{n}}+\cdots+\chi_{\Lambda_{m-1}^{n}}$. The resultant representation on the quotient space is thus $\chi_{\Lambda_{m}^{n}}$. The above form for the action of $B_{n}$ will be used in the succeeding sections, in which the representation is given on a space $V_{n, m}$ spanned by a set labelled by $\mathscr{U}_{\mathrm{w}}^{m}$ with relations as in Lemma 2.3.

2.2. The category of tangles. Let $\mathscr{O}_{n}$ be the set of maps

$$
\{1,2, \ldots, n\} \rightarrow\{+1,-1\}
$$

and $\mathscr{O}=\bigcup_{n=1}^{\infty} \mathscr{O}_{n}$. The category of tangles, $\mathbf{T}$, consists of the set of objects $\mathscr{O}$; and for any $a, b \in \mathscr{O}$, say $a \in \mathscr{O}_{m}, b \in \mathscr{O}_{n}$, the space $\operatorname{Morph}(a, b)$ consists of all oriented compact one-dimensional submanifolds, $M$, of $\mathbb{C} \times[0,1]$ for which,

$$
\partial M=-\sum_{i=1}^{m} a(i)(i, 0)+\sum_{j=1}^{n} b(j)(j, 1),
$$

while two such morphisms, $M$ and $M^{\prime}$, are considered to be equivalent if they are isotopic as submanifolds of $\mathbb{C} \times[0,1]$ with fixed boundary. The composition of morphisms $M_{1} \in \operatorname{Morph}(a, b)$ and $M_{2} \in \operatorname{Morph}(b, c)$ with $a, b, c \in \mathscr{O}$ is defined to be that morphism $N \in \operatorname{Morph}(a, c)$ specified by,

$$
N=\left\{(z, t / 2) \mid(z, t) \in M_{1}\right\} \cup\left\{(z,(1+t) / 2) \mid(z, t) \in M_{2}\right\} .
$$

An element of $\operatorname{Morph}(a, b)$ for any $a, b \in \mathscr{O}$ is called a tangle and may be pictured by using the projection of $\mathbb{C} \times[0,1]$ onto $\mathbb{R} \times[0,1]$, given by,

$$
(z, t) \mapsto(\mathfrak{R}(z), t)
$$

as a union of oriented curves lying between two parallel lines in the plane, joining $m$ points on one line to $n$ points on the other, with over and under crossing marked. An example is given in Figure 2. Thus $a(r)=+1$ corresponds to the curve having orientation $\uparrow$ at $(r, 0)$. 


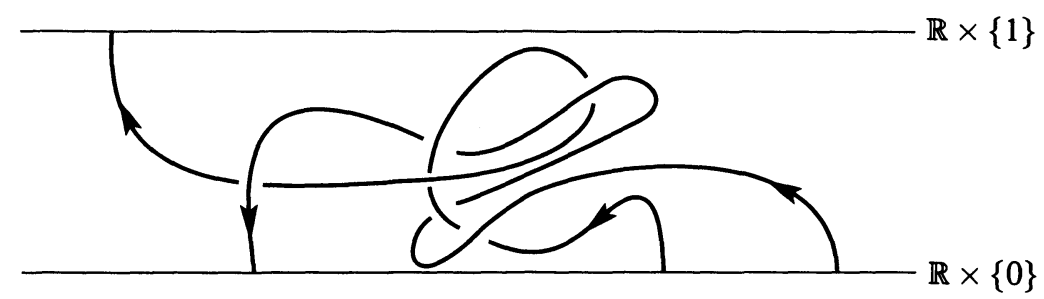

FIGURE 2

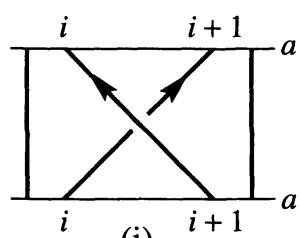

(i)

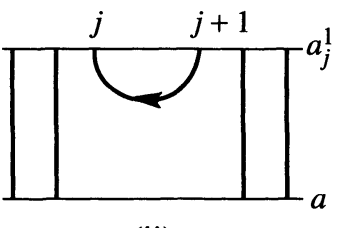

(ii)

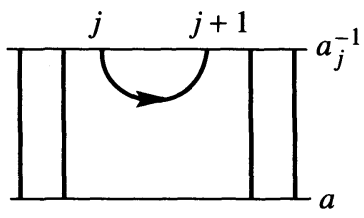

(iii)
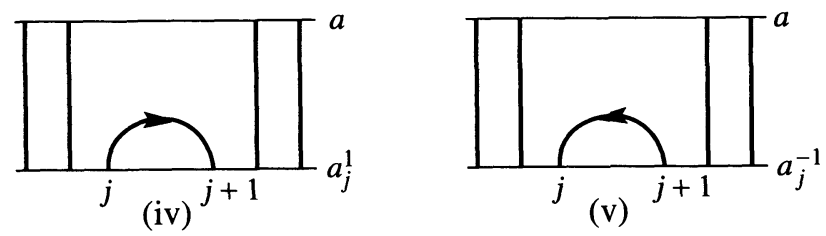

(v)

Figure 3

A presentation of the category of tangles first appeared in [14]. In [11] an alternative presentation was given, which we now recall. It is possible to express any tangle as a composition of elementary tangles, each being of one of the following forms.

(i) An element of $\operatorname{Morph}(a, a)$ for $a \in \mathscr{O}_{n}$ with $a_{i}=a_{i+1}=1$, in which there are $n$ curves, containing $\{j\} \times[0,1]$ for $j \in$ $\{1,2, \ldots, n\}, j \neq i, i+1$ (oriented according to $a(j))$, together with two upward moving strands which cross as shown in Figure 3(i).

(ii) Elements of $\operatorname{Morph}(a, b)$ for any $a \in \mathscr{O}_{n}$ and $b \in \mathscr{O}_{n+2}$ in which,

$$
b(i)= \begin{cases}a(i) & i<j, \\ a(i-2) & i>j+1, \\ \varepsilon & i=j, \\ -\varepsilon & i=j+1,\end{cases}
$$

for some $1 \leq j \leq n+1, \varepsilon= \pm 1$. Such $b$ is denoted by $a_{j}^{\varepsilon}$, and one may specify elements of $\operatorname{Morph}\left(a, a_{j}^{\varepsilon}\right), \operatorname{Morph}\left(a_{j}^{\varepsilon}, a\right)$ as shown in Figure $3(\mathrm{ii})-(\mathrm{v})$, all of which contain the $n$ lines 


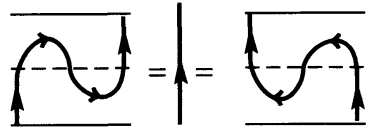

(a)

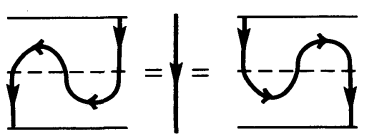

(b)

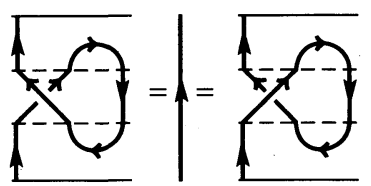

(c)
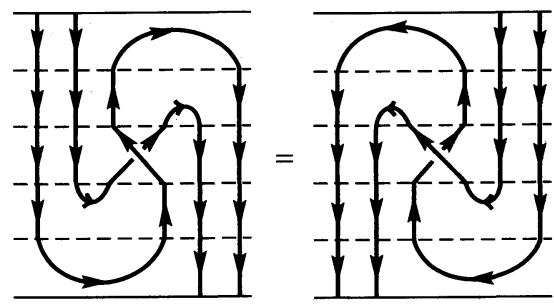

(d)

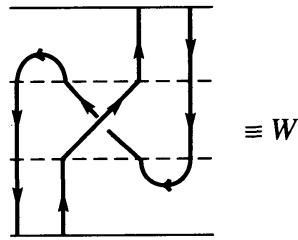

$\equiv W$

FIGURE 5

joining the points of $a$ to corresponding ones of $a_{j}^{\varepsilon}$ in $\{1,2, \ldots$, $n+2\} \backslash\{j, j+1\}$.

The relations satisfied by these generators are given by the diagrams in Figure 4; they should be thought of as relations connecting products of the above generators.

Theorem 2.4 (Turaev). The category of tangles is generated by the morphisms of Figure 3, for all $a \in \mathscr{O}_{n}, 1 \leq i \leq n, 1 \leq j \leq n+1$, together with the relations of Figure 4 along with the additional relations $E \circ W=W \circ E=I$, where $E$ and $W$ are the compositions of generators specified in Figure 5.

By a representation of the category of tangles is meant a map $\rho$, which assigns to each $a \in \mathscr{O}$ a vector space $V_{a}$, and to each $M \in \operatorname{Morph}(a, b)$ (where $a, b \in \mathscr{O}$ ), a linear transformation,

$$
\rho(M): V_{a} \rightarrow V_{b},
$$

such that composition of morphisms is respected. Any link $L$ in $\mathbb{R}^{3}$ can be represented by a projection onto a two-dimensional plane with overcrossings and under-crossing; that is, $L$ can be specified by an element 
of $\operatorname{Morph}(\varnothing, \varnothing)$ where $\varnothing$ denotes the single element of $\mathscr{O}_{0}$, called the vacuum. Hence any representation $\rho$ which is well defined (i.e., satisfies the relations of Theorem 2.4) defines an invariant of links as a map, $V_{\varnothing} \rightarrow$ $V_{\varnothing}$. In all the representations which we consider in this paper, $V_{\varnothing}=\mathbb{C}$, so that a linear transformation on $V_{\varnothing}$ is specified by the scaling factor. In this respect finding representations of the category of tangles may be viewed as a generalization of the problem of finding link invariants.

\section{Construction of representation}

In this section a representation of the category of tangles, $T$, will be defined geometrically. We will actually define a representation of the category, $\mathbb{C T}$, defined analogously to $\mathbf{T}$, except that now the objects are arbitrary configurations of distinct points in $\mathbb{C}$, with orientations. That is, $\mathscr{O}$ is replaced by $\mathbb{C} \mathscr{O}=\bigcup_{n=1}^{\infty}\left(X_{n} \times \mathscr{O}_{n}\right)$. There is a functor $\mathbf{T} \rightarrow \mathbb{C T}$ by which an object $a \in \mathscr{O}_{n}$ maps to $((1, \ldots, n), a) \in X_{n} \times \mathscr{O}_{n}$, so that any representation of $\mathbb{C T}$ gives rise to one of $\mathbf{T}$. The generators and relations for $\mathbb{C T}$ may be obtained analogously to those for $\mathbf{T}$ given in Theorem 2.4, and then the morphisms of Figure 3(i) are replaced by morphisms associated with arbitrary paths in $X_{n}$. For any $a \in \mathscr{O}_{n}$, let $\omega(a) \in \mathbb{N} \cup\{0\}$ denote the number of +1 's in the sequence $a(1), \ldots, a(n)$. For any object $A=\left(\left(w_{1}, \ldots, w_{n}\right), a\right)$ in $\mathbb{C T}$, let $\pi(A)$ denote the vector space $V_{n, \omega(a)}(\mathbf{w})$, the subspace of the fibre of $\widetilde{E}_{\omega(a)}(q)$ over $\mathbf{w} \in \widetilde{X}_{n}$ appearing in Lemma 2.3.

We shall construct a representation $\pi$, of the category $\mathbb{C T}$, in which objects $A$ are associated with the vector spaces $\pi(A)$. By the construction of $\widetilde{E}_{m}(q)$, there is a natural flat connection upon it, namely the Gauss-Manin connection which is defined once an $\left(S_{m} \times S_{n}\right)$-invariant local system on the total space of the initial fibration $X_{m+n} \rightarrow X_{n}$ is supplied; see $\S 2.1$. Parallel transport thus supplies the images under $\pi$ of the generators of $\mathbb{C T}$, whose type derives from Figure 3(i).

To complete the specification of $\pi$ it remains to define its action upon the generators of $\mathbb{C T}$ derived from Figures 3(ii)-(v). Suppose $A=\left(\left(w_{1}\right.\right.$, $\left.\left.\ldots, w_{n+2}\right), a\right)$ and $B=\left(\left(w_{1}^{\prime}, \ldots, w_{n}^{\prime}\right), b\right)$ with $a \in \mathscr{O}_{n+2}$ and $b \in$ $\mathscr{O}_{n}$, and that $\tau \in \operatorname{Morph}(A, B)$ in $\mathbb{C T}$ has type deriving from Figures 3(iv),(v). That is, the form of the horizontal slices, $(\mathbb{C} \times\{t\}) \cap \tau$ for $0 \leq t \leq 1$, of $\tau$, considered as a submanifold of $\mathbb{C} \times[0,1]$, can be described as follows for a suitable choice of $t_{0} \in(0,1)$ : 
(i) a set of $(n+2)$ distinct points $\left\{w_{i}(t) \mid 1 \leq i \leq n+2\right\}$ continuously parametrized by $t \in\left[0, t_{0}\right)$;

(ii) as $t \rightarrow t_{0}-$, two of these points, say $w_{j}(t)$ and $w_{j+1}(t)$, coalescing at $w_{0}$ say;

(iii) a set of distinct points $\left\{w_{i}^{\prime}(t) \mid 1 \leq i \leq n\right\}$ continuously parametrized by $t \in\left(t_{0}, 1\right]$;

while $w_{k}(0)=w_{k}, w_{j}^{\prime}(1)=w_{j}$ for all $j$ and $k, w_{i}\left(t_{0}\right)=w_{i}^{\prime}\left(t_{0}\right)$ for $i<\lambda$ and $w_{i}\left(t_{0}\right)=w_{i-2}^{\prime}\left(t_{0}\right)$ for $i>\lambda+1$. Lastly, $a \in \mathscr{O}_{n+2}$ and $b \in \mathscr{O}_{n}$ are related by $a=b_{j}^{\varepsilon}$, with $\varepsilon=+1$ in Figure 3(iv) or (v). Thus the action of $\pi$ on $\tau$ may be given as a map $V_{n+2, m+1}(\mathbf{w}) \rightarrow V_{n, m}\left(\mathbf{w}^{\prime}\right)$, where $m=\omega(b)=\omega(a)-1$. Parallel transport supplies natural isomorphisms $V_{n+2, m+1}(\mathbf{w}) \cong V_{n+2, m+1}\left(\mathbf{w}\left(t_{1}\right)\right)$ and $V_{n, m}\left(\mathbf{w}^{\prime}\right) \cong V_{n, m}\left(\mathbf{w}^{\prime}\left(t_{2}\right)\right)$ for any $0 \leq$ $t_{1}<t_{0}<t_{2} \leq 1$. Hence, without loss of generality, and to simplify the geometrical visualization of the construction, one may assume $w_{j}(t)$, for $j \neq i, i+1$ and $w_{j}^{\prime}(t)$, all to be independent of $t$.

As was discussed in $\S 2.1$, elements of $V_{n, m}$ may be considered as represented by functions $f: Y_{\mathbf{w}, m} \rightarrow \mathbb{C}$ which are holomorphic multi-valued functions twisting according to $\chi$. Suppose $\left[f_{t}\right]\left(0 \leq t<t_{0}\right)$ represents an element of $V_{n+2, m+1}(\mathbf{w}(t))$, where $f_{t}$ is a multi-valued holomorphic function on $Y_{\mathbf{w}(t), m+1} / S_{m+1}$ twisting according to $\chi$. Consider a path $\gamma_{t}$ defined for $t \in\left[0, t_{0}\right)$, in $Y_{\mathbf{w}, m+1}$, in which $z_{i}$ are fixed for $1 \leq i \leq m$ while $z_{m+1}$ follows a path with winding numbers $a(j), a(j+1)$ around $w_{j}, w_{j+1}$ and 0 around $w_{i}$ (any $\left.i \neq j, j+1\right)$. The only constraint on the choice of $\left\{\gamma_{t}\right\}$ is that it varies continuously with $t \in\left[0, t_{0}\right)$ and its diameter tends to zero as $t \rightarrow t_{0}-$. Then, $\int_{\gamma_{t}} f_{t} d z_{m+1}$ defines a multivalued holomorphic function on $Y_{\mathbf{w}(t), m}$, whose limit as $t \rightarrow t_{0}-$ provides a multi-valued holomorphic function $g$ on $Y_{\mathbf{w}^{\prime}\left(t_{0}\right), m}$. That there is no remaining singularity at $w_{0}$ can be seen by noting that the monodromy of $g$ when $z_{j}$ goes around $w_{0}$ has contributions from the twists around all three points $w_{i}, w_{i+1}$ and $z_{m+1}$, while,

$$
q_{z_{j} w_{i}} q_{z_{j} z_{m+1}} q_{z_{j} w_{i+1}}=1 \text {. }
$$

From its definition it is apparent that $g$ represents an element of $V_{n, m}$, and hence this establishes a map $V_{n+2, m+1}\left(\mathbf{w}\left(t_{0}-\right)\right) \rightarrow V_{n, m}\left(\mathbf{w}^{\prime}\left(t_{0}+\right)\right)$, the corresponding total map $V_{m+2, m+1}(\mathbf{w}) \rightarrow V_{n, m}\left(\mathbf{w}^{\prime}\right)$ defining $\pi(\tau)$. This action of $\pi(\tau)$ may be formally written as follows,

$$
f \mapsto \lim \left(\oint_{\infty} f d z_{m+1}\right)=g
$$



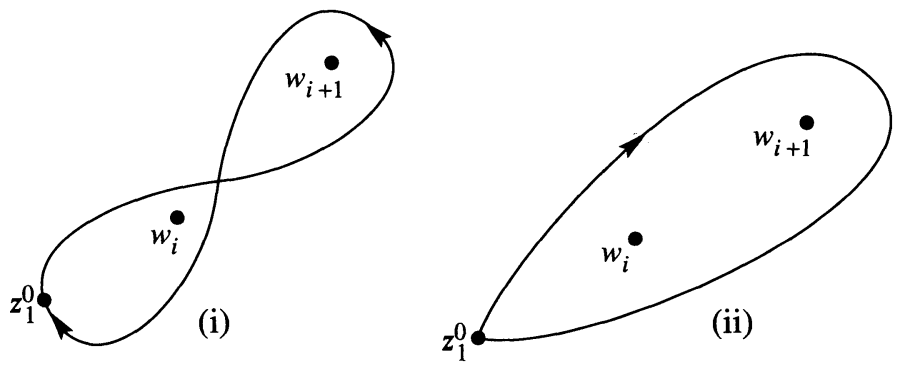

FIGURE 6

The definition of the image under $\pi$ of morphisms $\tau$ whose type derives from Figures (ii), (iii) similarly reduces to the problem of defining a suitable map $V_{n, m}\left(\mathbf{w}^{\prime}\left(t_{0}+\right)\right) \rightarrow V_{n+2, m+1}\left(\mathbf{w}\left(t_{0}-\right)\right)$ in the opposite direction to that just considered. Up to scaling, it is an inverse of the preceding map. Once a base-point is chosen, such an inverse may be fixed in terms of representative functions by mapping $g$ to a multi-valued holomorphic function $f$, satisfying $(*)$, for which,

$$
\int_{\bigcirc} f d z_{m+1}=0
$$

where the integral is taken around the path followed by $z_{1}$ with base-point $z_{1}^{0}$ and winding numbers -1 around both $w_{i}$ and $w_{i+1}$. See Figure 6 for the two paths $\infty$ and $\bigcirc$. Note that since the former loop lifts to a loop in $\widetilde{Y}_{w, m}$, there is no necessity to fix a base-point for the curve.

For the case of a trivial twisting, that is $q=1, V_{n, m}(\mathbf{w})$ corresponds to the space of those (single-valued) holomorphic functions on $Y_{w, m}$, of the form,

where

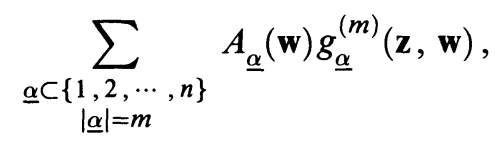

$$
g_{\underline{\alpha}}^{(m)}(\mathbf{z}, \mathbf{w})=\sum_{\sigma \in S_{m}} \prod_{i=1}^{m}\left(z_{\sigma(i)}-w_{\alpha_{i}}\right)^{-1}
$$

for $\underline{\alpha}=\left\{\alpha_{1}, \ldots, \alpha_{m}\right\} \subset\{1,2, \ldots, n\}$. The Gauss-Manin connection gives a parallel transport in which a section is flat $\Leftrightarrow\left\{A_{\underline{\alpha}}(\mathbf{w})\right\}$ are independent of $\mathbf{w}$, for all $\underline{\alpha}$. The action of $B_{n}$ reduces to the natural action of $S_{n}$ on subsets $\underline{\alpha}$ of $\{1,2, \cdots, n\}$ whose order is $m$, so that the monodromy representation is $\operatorname{Ind}_{S_{m}}^{S_{n}}(1)$. Morphisms of types (iv) and (v) 
act by integration around the figure-8 loop of Figure 6(i),

$$
g=\lim _{t \rightarrow t_{0}-}\left[ \pm \operatorname{Res}_{z_{m+1}}\left(f, w_{j+1}\right) \mp \operatorname{Res}_{z_{m+1}}\left(f, w_{j}\right)\right] .
$$

For example, $f=g_{\underline{\alpha}}^{(m+1)}$ transforms to 0 unless $\left.\mid \underline{\alpha} \cap\{i, i+1)\right\} \mid=1$, in which case the image is $\pm g_{\underline{\beta}}^{(m)}$ for $\underline{\alpha}=\underline{\beta} \cup\{i+1\}$ and $\mp g_{\underline{\beta}}^{(m)}$ for $\underline{\alpha}=\underline{\beta} \cup\{i\}$, where $|\beta \cap\{i, i+\underline{1}\}|=0$.

Recall that $V_{n, m}$ consists of those homology classes associated with (multi-valued) holomorphic functions, $f$, twisting according to $\chi$, for which,

$$
\int_{\gamma_{\underline{\alpha}}} f=0 \quad \forall \underline{\alpha} \in \mathscr{S}_{\mathbf{w}}^{m} \backslash \mathscr{T}_{\mathbf{w}}^{m}
$$

while being invariant under the action of $S_{m}$. Relative to the basis $\left\{f_{\underline{\alpha}}\right\}$ defined dual to chains $\left\{\gamma_{\underline{\alpha}}\right\}$ (see $\S 2.1$ ), the linear transformations $V_{A} \stackrel{\underline{\alpha}}{\rightarrow}$ $V_{B}$ determined by the images under $\pi$ of the elements of $\operatorname{Morph}(A, B)$ derived from Figure 3(i)-(v) are expressible as follows:

$$
f_{\underline{\alpha}} \mapsto \begin{cases}q^{1 / 2}\left((1-q) f_{\underline{\beta} w_{j}}+f_{\underline{\beta} w_{j+1}}\right) & \text { if } \underline{\alpha}=\underline{\beta} w_{j}, \\ q^{1 / 2} \cdot q f_{\underline{\beta} w_{j}} & \text { if } \underline{\alpha}=\underline{\beta} w_{j+1}, \\ q^{1 / 2} \cdot f_{\underline{\alpha}} & \text { otherwise ; }\end{cases}
$$

(ii), (iii) $\quad f_{\underline{\alpha}} \mapsto \pm q^{r-j / 2}\left(-q^{-1} f_{\underline{\alpha} w_{j+1}}+f_{\underline{\alpha} w_{j}}\right)$;

(iv), (v) $\quad f_{\underline{\alpha}} \mapsto \begin{cases} \pm q^{(j+1) / 2-r} f_{\underline{\beta}} & \text { if } \underline{\alpha}=\underline{\beta} w_{j}, \\ \mp q^{(j+1) / 2-r} f_{\underline{\beta}} & \text { if } \underline{\alpha}=\underline{\beta} w_{j+1}, \\ 0 & \text { otherwise; }\end{cases}$

where $r$ denotes the number of $\alpha_{i}$ 's less than $j$; and in all the expressions $\left\{\alpha_{i}\right\}$ are distinct elements of $\left\{w_{1}, \ldots, w_{n}\right\}$ while $f_{\underline{\beta} w_{i}}$ refers to $f_{\underline{\gamma}}$ when $\underline{\gamma} \in \mathscr{U}_{w}^{m}$ is $\underline{\beta} w_{i}$ reordered so as to be in decreasing sequence. These formulae can easily be seen to agree with the geometric definitions given above, certainly in the case of $q=1$.

Lemma 3.1. The above definitions give rise to well-defined maps $V_{n, m} \rightarrow$ $V_{n, m}, V_{n, m} \rightarrow V_{n+2, m+1}$ and $V_{n+2, m+1 L} \rightarrow V_{n, m}$.

Proof. This lemma is proved by checking that the relations of Lemma 2.3 are preserved. Since (i) is derived from the natural monodromy action, it preserves the boundary space, and thus also the relation of Lemma 2.3. 


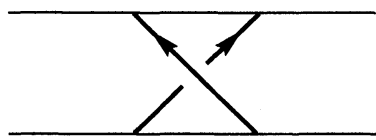

(i)

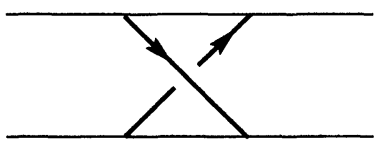

(iii)

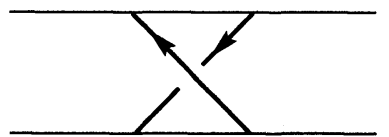

(ii)

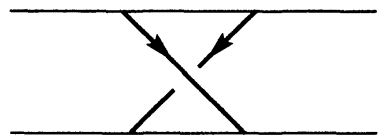

(iv)

\section{FiguRe 7}

Under (ii), the 1.h.s. of the relation in Lemma 2.3 transforms to

$$
\begin{aligned}
& \sum_{\substack{j<i \\
w_{j} \notin \underline{\beta}}} q^{s_{j}} q^{r+1-i / 2}\left(q^{-1} f_{\underline{\beta} w_{j} w_{i+1}}-f_{\underline{\beta} w_{j} w_{i}}\right) \\
& +\sum_{\substack{j>i+1 \\
w_{j} \notin \underline{\beta}}} q^{s_{j}} q^{r-i / 2}\left(q^{-1} f_{\underline{\beta} w_{j} w_{i+1}}-f_{\underline{\beta} w_{j} w_{i}}\right),
\end{aligned}
$$

where $\underline{\beta}$ is a sequence of $m-1$ distinct elements of $\left\{w_{1}, \ldots, w_{i-1}\right.$, $\left.w_{i+2}, \ldots, w_{n+2}\right\}$. This is a combination of boundaries in $V_{n+2, m+1}$ associated with the sequences $\underline{\beta} w_{i+1}, \underline{\beta} w_{i}$ of $m$ elements in $\left\{w_{1}, \ldots, w_{n+2}\right\}$. It may similarly be checked that (iii), (iv) and (v) preserve the relations of Lemma 2.3. q.e.d.

It may also be verified that the following lemma holds.

Lemma 3.2. The representation $\pi$ of the generators of the category of tangles defined above satisfies all the relations of Theorem 2.4.

Thus $\pi$ defines a representation of the category of tangles. The images of the morphisms of Figure 7 may be computed in terms of the generators, and the results are given below.

$\begin{aligned} & \text { (i), (iv) } f_{\underline{\alpha}} \mapsto \begin{cases}q^{1 / 2}(1-q) f_{\underline{\alpha}}+q^{1 / 2} f_{\underline{\alpha}_{i}} & \text { if } w_{i} \in \underline{\alpha}, w_{i+1} \notin \underline{\alpha}, \\ q^{3 / 2} \cdot f_{\underline{\alpha}_{i}} & \text { if } w_{i} \notin \underline{\alpha}, w_{i+1} \in \underline{\alpha}, \\ q^{1 / 2} \cdot f_{\underline{\alpha}} & \text { otherwise ; }\end{cases} \\ & \text { (ii), (iii) } \quad f_{\underline{\alpha}} \mapsto \begin{cases}f_{\underline{\alpha}_{i}} & \text { if } w_{i} \notin \underline{\alpha}, w_{i+1} \in \underline{\alpha}, \\ \left(q^{-1}-1\right) f_{\underline{\alpha}}+q^{-1} f_{\underline{\alpha}_{i}} & \text { if } w_{i} \in \underline{\alpha}, w_{i+1} \notin \underline{\alpha}, \\ q^{-1} f_{\underline{\alpha}} & \text { otherwise. }\end{cases} \end{aligned}$ 


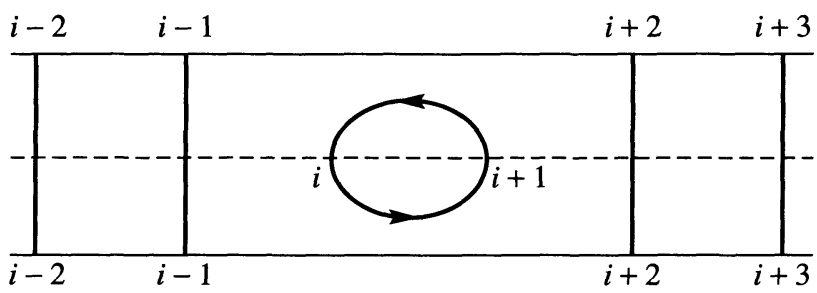

FIGURE 8

The representation $\pi$ gives rise to an invariant of tangles in the form of an operator. As discussed in $\S 2.2$, and since $V_{\varnothing}=V_{0,0}=\mathbb{C}$, it gives a complex number invariant of links. Since the pairs of morphisms (i), (iv) and (ii), (iii) transform under $\pi$ to pairs of equal operators, this invariant is unaltered under the reversal of the orientations of all components of the link. When the link $L$ is replaced by the union of $L$ with a single unknotted and unlinked component, the invariant scales up by $q^{1 / 2}+$ $q^{-1 / 2}$. For, under $\pi$, the morphism in $\operatorname{Morph}(a, a)$ shown in Figure 8 transforms to a composition of two maps,

$$
\begin{aligned}
f_{\underline{\alpha}} & \mapsto q^{r-i / 2}\left(q^{-1} f_{\underline{\alpha} w_{i+1}}-f_{\underline{\alpha} w_{i}}\right) \\
& \mapsto q^{r-i / 2}\left(q^{-1} \cdot q^{(i+1) / 2-r} f_{\underline{\alpha}}-\left(-q^{(i+1) / 2-r} f_{\underline{\alpha}}\right)\right) \\
& =\left(q^{1 / 2}+q^{-1 / 2}\right) f_{\underline{\alpha}} .
\end{aligned}
$$

Denote the invariant so produced by $P(L)$. Then consider three links $L_{+}, L_{-}$and $L_{0}$, which differ at one crossing as shown in Figure 9. The three tangles transform under $\pi$ into maps which take $f_{\underline{\alpha}}$ to,

$$
\begin{array}{cccc}
q^{-1 / 2} f_{\underline{\alpha}}, & q^{1 / 2} f_{\underline{\alpha}}, & f_{\underline{\alpha}}, & \text { if } w_{i}, w_{i+1} \in \underline{\alpha} \\
q^{-3 / 2} f_{\underline{\alpha}_{i}}, & q^{1 / 2}\left((1-q) f_{\underline{\alpha}}+f_{\underline{\alpha}_{i}}\right) & f_{\underline{\alpha}}, & \text { if } w_{i} \in \underline{\alpha}, w_{i+1} \notin \underline{\alpha} ; \\
q^{-1 / 2}\left(f_{\underline{\alpha}_{i}}+\left(1-q^{-1}\right) f_{\underline{\alpha}}\right), & q^{3 / 2} f_{\underline{\alpha}_{i}}, & f_{\underline{\alpha}}, & \text { if } w_{i} \notin \underline{\alpha}, w_{i+1} \in \underline{\alpha} ;
\end{array}
$$

respectively. It may be noted that these images satisfy a linear relation irrespective of whether $w_{i}$ and $w_{i+1}$ lie in $\underline{\alpha}$. Hence,

$$
q P\left(L_{+}\right)-q^{-1} P\left(L_{-}\right)+\left(q^{-1 / 2}-q^{1 / 2}\right) P\left(L_{0}\right)=0 .
$$

Note also that the same relation is obtained between the linear transformations associated with the three tangles of Figure 10. This is predictable, as it follows from the invariance of the number $P(L)$ on the class of links. However the Jones polynomial $X_{L}(q, \lambda)$ satisfies the skein relation,

$$
q^{1 / 2} \lambda^{1 / 2} X_{L_{+}}(q, \lambda)+\left(q^{-1 / 2}-q^{1 / 2}\right) X_{L_{0}}(q, \lambda)-q^{-1 / 2} \lambda^{-1 / 2} X_{L_{-}}(q, \lambda)=0 \text {, }
$$




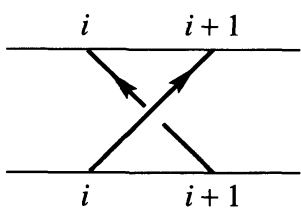

$L_{+}$

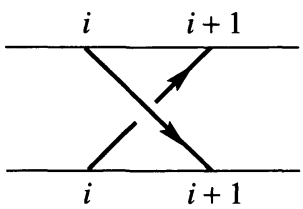

$L_{+}$

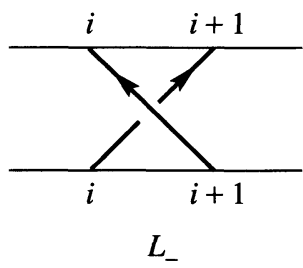

Figure 9

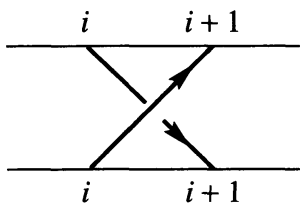

L
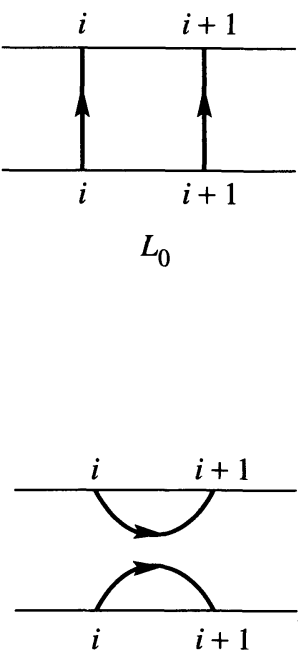

$L_{0}$

Figure 10

while $X_{L}(q, \lambda)=1$ whenever $L$ is the one-component unknot. The following connection between $P(L)$ and $X_{L}(q, q) \equiv V_{L}(q)$ is thus obtained.

Theorem 3.3. The invariant of links defined by the representation $\pi$ of the category of tangles is $\left(q^{1 / 2}+q^{-1 / 2}\right) V_{L}(q)$, a multiple of the one-variable Jones polynomial.

The extra factor $\left(q^{1 / 2}+q^{-1 / 2}\right)$ appearing here is due to the nonfunctionality of the standard normalization of $V_{L}$; it is necessary to make $V_{L}$ multiplicative with respect to disjoint union, and thus expressible in a functorial manner. There is an essentially unique representation of the category of tangles giving $V_{L}$ as the corresponding link invariant. In particular, the above representation of $\mathbf{T}$, obtained geometrically, is equivalent to that obtained by other known procedures, for example those of [9] from an appropriate Hopf algebra, or of [13] using topological quantum field theory. This remark holds good for generic $q$, that is, away from those roots of unity with 'small' order. For roots of unity which have order less than $n$, the homological construction of $V_{n, m}(\mathbf{w})$, using say the limiting lemma of [5], gives a vector space whose dimension may be greater than $\left(\begin{array}{l}n \\ m\end{array}\right)-\left(\begin{array}{c}n \\ m-1\end{array}\right)$, whilst the 'true' dimension, as constructed by other approaches, will actually be less than this generic value. To this extent, our geometrically defined functor differs from that of [9], whilst still giving $V_{L}$ on links. 


\section{Braid approach}

Let $\pi_{\Lambda_{m}^{n}}$ denote the representation of $H_{n}(q)$ associated with the tworow Young diagram $\Lambda_{m}^{n}$. Denote the associated character by $\chi_{n, m}$. In the limit $q \rightarrow 1, \pi_{\Lambda_{m}^{n}}$ tends to the standard representation of $S_{n}$ associated with $\Lambda_{m}^{n}$. In this limit, for all $\sigma \in S_{m}$,

$$
\chi_{n, m}(\sigma)=\sigma^{m}-\sigma^{m-1}
$$

where $\sigma^{m}$ denotes the number of ways $m$ can be expressed as a sum of lengths of cycles in $\sigma$.

Lemma 4.1. In the limit $q \rightarrow 1$,

$$
\chi_{2 n, n}\left(\sigma_{1} \otimes \sigma_{2}\right)=\sum_{r=0}^{[n / 2]} \chi_{n, r}\left(\sigma_{1}\right) \chi_{n, r}\left(\sigma_{2}\right) \text { for any } \sigma_{1}, \sigma_{2} \in S_{n} .
$$

In this lemma, $\sigma_{1} \otimes \sigma_{2}$ denotes the element of $S_{2 n}$ given by the permutation $\sigma_{1}$ on $\{1,2, \ldots, n\}$ and $\sigma_{2}$ on $\{n+1, \ldots, 2 n\}$. It may be deduced that for general $q$, the restriction of $\pi_{\Lambda_{n}^{2 n}}$ to $H_{n}(q) \times H_{n}(q) \subset H_{2 n}(q)$ may be decomposed as in the following theorem.

Theorem 4.2. The restriction of the representation $\pi_{\Lambda_{n}^{2 n}}$ of $H_{2 n}(q)$ to $H_{n}(q) \times H_{n}(q)$ may be decomposed as a direct sum of the representations $\pi_{\Lambda_{r}^{n}} \otimes \pi_{\Lambda_{r}^{n}}$ over $r=0,1, \ldots,[n / 2]$.

Example. An explicit realisation of the isomorphism is now given when $n=3$. The representation $\pi_{\Lambda_{m}^{n}}$ is considered on $V_{n, m}$ with spanning set $\left\{f_{\underline{\alpha}} \mid \underline{\alpha} \in \mathscr{U}_{\mathrm{w}}^{m}\right\}$. A suitable basis consists of those $\underline{\alpha} \in \mathscr{U}_{\mathrm{w}}^{m}$ for which $\alpha_{r} \geq 2(m+1-r) \forall r$. Such $\underline{\alpha}$ may be put in 1-1 correspondence with standard Young tableaux of shape $\Lambda_{m}^{n}$, that is, an assignment of $\{1,2, \ldots, n\}$ to the squares in $\Lambda_{m}^{n}$ in such a way that reading from left to right or top to bottom along any row or column, yields an increasing sequence. Thus in the case $n=5, m=2$, we have $\left|\mathscr{U}_{\mathrm{w}}^{m}\right|=10$ and $\left|\mathscr{U}_{\mathrm{w}}^{m-1}\right|=5$ and so $V_{5,2}$ may be expressed in terms of a spanning set of 10 elements with 5 relations (see Lemma 2.3). Alternatively the basis $\left(f_{\underline{\alpha}}\right)$ may be used, in which the only allowed $\underline{\alpha}=\left(\alpha_{1}, \alpha_{2}\right)$ are,

$$
\left(w_{5}, w_{4}\right),\left(w_{5}, w_{3}\right),\left(w_{4}, w_{3}\right),\left(w_{5}, w_{2}\right),\left(w_{4}, w_{2}\right) \text {. }
$$

They correspond to the five standard tableaux on $\Lambda_{2}^{5}$ shown below.
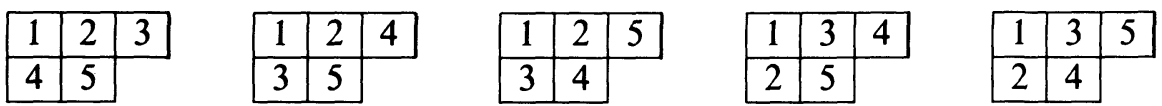
The $\underline{\alpha}$ associated to a Young tableau is obtained by reading off the elements assigned to the squares in the second row, from right to left. Using this notation, $V_{3,0}$ and $V_{3,1}$ are found to have dimensions 1 and 2, with bases specified by the tableaux,
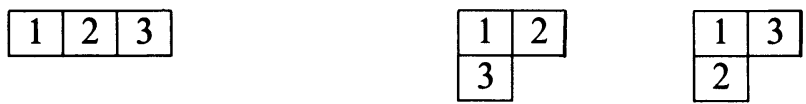

respectively, while $V_{6,3}$ is 5 -dimensional with associated tableaux as shown below.

\begin{tabular}{|l|l|l|}
\hline 1 & 2 & 3 \\
\hline 4 & 5 & 6 \\
\hline
\end{tabular}

\begin{tabular}{|l|l|l|}
\hline 1 & 2 & 4 \\
\hline 3 & 5 & 6 \\
\hline
\end{tabular}

\begin{tabular}{|l|l|l|}
\hline 1 & 3 & 4 \\
\hline 2 & 5 & 6 \\
\hline
\end{tabular}

\begin{tabular}{|l|l|l|}
\hline 1 & 2 & 5 \\
\hline 3 & 4 & 6 \\
\hline
\end{tabular}

\begin{tabular}{|l|l|l|}
\hline 1 & 3 & 5 \\
\hline 2 & 4 & 6 \\
\hline
\end{tabular}

Denote the associated bases for $V_{3,0}, V_{3,1}$ and $V_{6,3}$ by $\{\mathbf{f}\},\left\{\mathbf{f}_{1}, \mathbf{f}_{2}\right\}$ and $\left\{\mathbf{e}_{1}, \ldots, \mathbf{e}_{5}\right\}$, respectively. The isomorphism of Theorem 4.2 is now given by Table 1 , where $\lambda$ is arbitrary.

TABLE 1

\begin{tabular}{r|ccccc} 
& $\mathbf{e}_{1}$ & $\mathbf{e}_{2}$ & $\mathbf{e}_{3}$ & $\mathbf{e}_{4}$ & $\mathbf{e}_{5}$ \\
\hline $\mathbf{f} \otimes \mathbf{f}$ & $1+q^{-1}+q^{-2}$ & -1 & $-q^{-1}$ & $-q^{-1}$ & $-q^{-2}$ \\
$\mathbf{f}_{1} \otimes \mathbf{f}_{1}$ & 0 & $q \lambda$ & 0 & $(1-q) \lambda$ & 0 \\
$\mathbf{f}_{1} \otimes \mathbf{f}_{2}$ & 0 & 0 & 0 & $\lambda$ & 0 \\
$\mathbf{f}_{2} \otimes \mathbf{f}_{1}$ & 0 & $q \lambda$ & $-q \lambda$ & $(1-q) \lambda$ & $(q-1) \lambda$ \\
$\mathbf{f}_{2} \otimes \mathbf{f}_{2}$ & 0 & 0 & 0 & $\lambda$ & $-\lambda$
\end{tabular}

We now return to the general case. Suppose $L$ is a link, expressed as the braid closure of $\beta \in B_{n}$. Then as a tangle, $L$ can be expressed as the element of $\operatorname{Morph}(\varnothing, \varnothing)$ given by,

$$
\gamma_{n}^{\prime}(\beta \otimes 1) \gamma_{n},
$$

where $\gamma_{n} \in \operatorname{Morph}\left(\varnothing, a_{n}\right), \gamma_{n}^{\prime} \in \operatorname{Morph}\left(a_{n}, \varnothing\right)$ and $\beta \otimes 1 \in \operatorname{Morph}\left(a_{n}, a_{n}\right)$. The object $a_{n} \in \mathscr{O}_{2 n}$ is the object in the category of tangles defined by,

$$
a_{n}(i)=\operatorname{sgn}(n+1 / 2-i),
$$

for $1 \leq i \leq 2 n$; while $\beta \otimes 1$ denotes the morphism of $2 n$ strings, the first $n$ braided according to $\beta$, with the last $n$ going downwards and being 


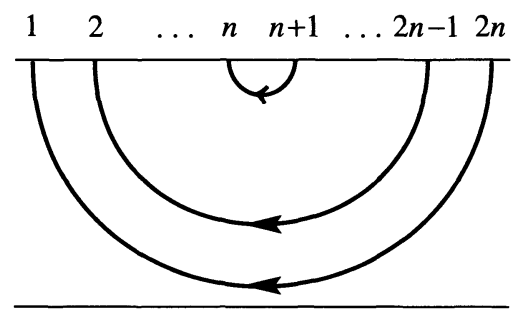

$\gamma_{n}$

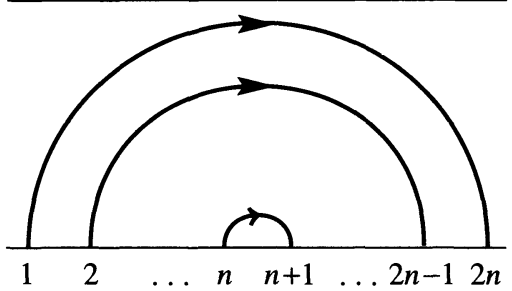

$\gamma_{n}^{\prime}$

FIGURE 11

lines joining $(r, 1)$ to $(r, 0)$ (for $n+1 \leq r \leq 2 n$ ). The morphisms $\gamma_{n}$ and $\gamma_{n}^{\prime}$ are illustrated in Figure 11. The representation $\pi$ assigns $V_{2 n, n}$ to objects $a_{n} \in \mathscr{O}$, while it is clear that the action of $\beta \otimes 1$ on $V_{2 n, n}$ is given by $\pi_{\Lambda_{n}^{2 n}}$, factoring through $H_{2 n}(q)$. Theorem 4.2 decomposes this action into,

$$
\bigoplus_{r=0}^{[n / 2]} \pi_{\Lambda_{r}^{n}}(\beta) \otimes \mathrm{id}
$$

If we denote the image of $\gamma_{n}$ in $V_{2 n, n} \cong \bigoplus_{r=0}^{[n / 2]} V_{n, r} \otimes V_{n, r}$ by $\sum_{r=0}^{[n / 2]} \sum_{i} v_{r}^{i} \otimes w_{r}^{i}$, then the invariant obtained is

$$
\begin{aligned}
P(L) & =\left\langle\sum_{r, i} v_{r}^{i} \otimes w_{r}^{i} \mid \sum_{r, i} \pi_{\Lambda_{r}^{n}}\left(v_{r}^{i} \otimes w_{r}^{i}\right)\right\rangle \\
& =\sum_{r} \sum_{i, j}\left\langle v_{r}^{i} \mid \pi_{\Lambda_{r}^{n}}\left(v_{r}^{j}\right)\right\rangle \cdot\left\langle w_{r}^{i} \mid w_{r}^{j}\right\rangle,
\end{aligned}
$$

where $\langle\mid\rangle$ denotes an inner product defined on $V_{2 n, n}$, so as to make $\gamma_{n}$ and $\gamma_{n}^{\prime}$ adjoints. However, in [3] V. Jones gives an expansion of $X_{L}(q, \lambda)$ in terms of Hecke algebra characters. This reduces in the case $\lambda=q$ to,

$$
V_{L}(q)=(\sqrt{q})^{e-n-1}(-1)^{n-1}\left(1-q^{2}\right)^{-1} \sum_{m=0}^{[n / 2]}\left(q^{m}-q^{n-m+1}\right) \chi_{\Lambda_{m}^{n}(\beta)} .
$$

Theorem 3.3 allows a comparison of (4.1) and (4.2).

Example. For links with braid number 3, i.e., $L=\widehat{\beta}$ for some $\beta \in B_{3}$, (4.2) reduces to,

$$
V_{L}(q)=(\sqrt{q})^{e}\left(\left(q+q^{-1}\right) \chi_{\Lambda_{0}^{3}}(\beta)+\chi_{\Lambda_{1}^{3}}(\beta)\right) .
$$


In the limiting case $q=1$, the correct form of (4.1) may be explicitly determined. The vector representing $\gamma_{3}$ is $\mathbf{v}_{0}=(0,2,1,1,2)^{\mathrm{T}}$ in terms of the basis $\mathbf{e}_{i}$ for $V_{6,3}$. Under the isomorphism with $V_{3,0}^{\otimes 2} \oplus V_{3,1}^{\otimes 2}$, discussed above,

$$
\mathbf{v}_{0} \mapsto(-2,2 \lambda, \lambda, \lambda,-\lambda)^{\mathrm{T}}
$$

while the inner product matrix has the form,

$$
\begin{gathered}
\mathbf{f} \otimes \mathbf{f} \\
\mathbf{f}_{1} \otimes \mathbf{f}_{1} \\
\mathbf{f}_{1} \otimes \mathbf{f}_{2} \\
\mathbf{f}_{2} \otimes \mathbf{f}_{1} \\
\mathbf{f}_{2} \otimes \mathbf{f}_{2}
\end{gathered} \quad\left(\begin{array}{ccccc}
3 & 0 & 0 & 0 & 0 \\
0 & 8 / 3 \lambda^{2} & -4 / 3 \lambda^{2} & -4 / 3 \lambda^{2} & 2 / 3 \lambda^{2} \\
0 & -4 / 3 \lambda^{2} & 8 / 3 \lambda^{2} & 2 / 3 \lambda^{2} & -4 / 3 \lambda^{2} \\
0 & -4 / 3 \lambda^{2} & 2 / 3 \lambda^{2} & 8 / 3 \lambda^{2} & -4 / 3 \lambda^{2} \\
0 & 2 / 3 \lambda^{2} & -4 / 3 \lambda^{2} & -4 / 3 \lambda^{2} & 8 / 3 \lambda^{2}
\end{array}\right) .
$$

Hence,

$$
\begin{aligned}
P(L)= & \left\langle\mathbf{v}_{0}|\pi(\beta \otimes 1)| \mathbf{v}_{0}\right\rangle \\
= & \left\langle\mathbf{v}_{0}\right|-2 \pi_{0}(\mathbf{f}) \otimes \mathbf{f}+2 \lambda \pi_{1}\left(\mathbf{f}_{1}\right) \otimes \mathbf{f}_{1}+\lambda \pi_{1}\left(\mathbf{f}_{1}\right) \otimes \mathbf{f}_{2} \\
& \left.+\lambda \pi_{1}\left(\mathbf{f}_{2}\right) \otimes \mathbf{f}_{1}-\lambda \pi_{1}\left(\mathbf{f}_{2}\right) \otimes \mathbf{f}_{2}\right\rangle
\end{aligned}
$$

which is seen to reduce to $12 \operatorname{tr} \pi_{0}+6 \operatorname{tr} \pi_{1}$. Here $\pi_{0}$ and $\pi_{1}$ denote the actions of $\pi_{\Lambda_{0}^{3}}(\beta)$ and $\pi_{\Lambda_{1}^{3}}(\beta)$ upon $\langle\mathbf{f}\rangle=V_{3,0}$ and $\left\langle\left\{\mathbf{f}_{1}, \mathbf{f}_{2}\right\}\right\rangle=V_{3,1}$. Note that this gives $P$ as a combination of the characters $\chi_{\Lambda_{0}^{3}}(\beta)$ and $\chi_{\Lambda_{1}^{3}(\beta)}$, and the ratio in which they occur is $2: 1$. This agrees with the ratio $\left(q+q^{-1}: 1\right)$ observed in (4.3).

In this section we have compared the expression for $V$ as a combination of Hecke algebra characters associated with 2-row Young diagrams, with that obtained from the tangle picture. Theorem 4.2 provides the link between the two approaches. In the next section we look briefly at other ways in which braids may be related to links, via plait closure.

\section{The plait approach}

Suppose $\beta$ is a braid on $2 n$ strings. It may be closed to form a link $L$ by joining the $2 n$ end-points, at one end, to each other, in $n$ adjacent pairs, and similarly for the points at the other end. This is pictured in Figure 12. The invariant $P(L)$ may thus be written as,

$$
\left\langle u_{n}^{\prime}|\pi(\beta)| u_{n}\right\rangle
$$




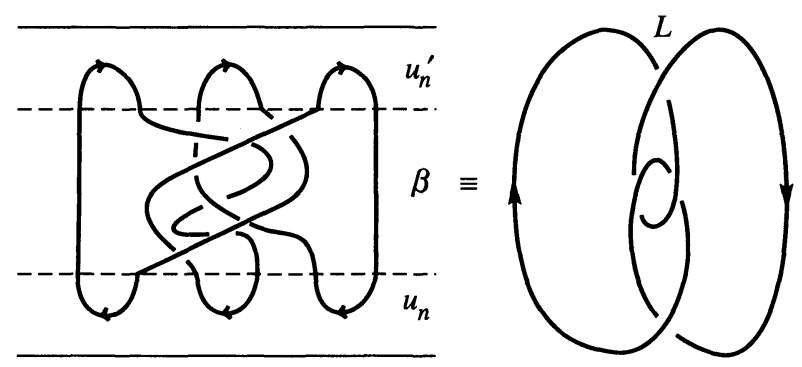

FIGURE 12

where $\left\langle u_{n}^{\prime}\left|\in \operatorname{Morph}\left(b_{n}, \varnothing\right),\right| u_{n}\right\rangle \in \operatorname{Morph}\left(\varnothing, b_{n}\right), \pi(\beta) \in \operatorname{Morph}\left(b_{n}, b_{n}\right)$. Here $b_{n}$ denotes the element of $\mathscr{O}_{2 n}$ with alternating signs, and $u_{n}, u_{n}^{\prime}$ are given by the bottom and top parts of Figure 12, respectively.

It is comparatively easy to describe $\beta, u_{n}, u_{n}^{\prime}$ topologically. The braid $\beta$ gives a tangle with $2 n$ strings, $n$ going upwards and $n$ going downwards. The action of $\beta$ on $V_{2 n, n}$ is the natural action induced by parallel transport. The element $\left|u_{n}\right\rangle$ of $V_{2 n, n}$ is defined, up to scaling, so that it corresponds to a function $f\left(z_{1}, \ldots, z_{n}\right)$ with singularities at $w_{1}, \ldots, w_{2 n}$ so that the integral around a loop containing $w_{2 j-1}$ and $w_{2 j}$ vanishes for $1 \leq j \leq n$. Similarly, $\left\langle u_{n}^{\prime}\right|$ is the functional on $V_{2 n, n}$, given by integrating around all $n$ figure- 8 loops around adjacent pairs $w_{2 j-1}, w_{2 j}$. This defines $\left\langle u_{n}^{\prime}\right|$ and $\left|u_{n}\right\rangle$ up to a scaling, and hence gives some form of topological definition for $V_{L}$. Note that it is not necessary to project into a plane in order to use this definition. In terms of $L \subseteq S^{3}$, pick a unit vector $\mathbf{e}$, and then investigate local maxima and minima of $\gamma(t) \cdot \mathbf{e}$. Deform $\gamma$ so that all local maxima have $\gamma(t) \cdot \mathbf{e}>N$ and similarly for local minima ( $N$ large). Alternatively subdivide $S^{3}$ by a 2-surface $\Sigma$ so that all local maxima occur on one side of $\Sigma$, and local minima on the other side; see Figure 13.

Then $V_{L}$ is the matrix element of the action of the braid connecting the local maxima and minima, on the middle-dimensional cohomology $H^{n}\left(Y_{2 n, n}\right)$, between the vectors given by functions anti-symmetric under the interchanges between pairs of points associated with the same local maxima/minima. The surface $\Sigma$ cuts $L$ into two halves. Each half joins the $2 n$ points $L \cap \Sigma=\left\{w_{1}, \cdots, w_{2 n}\right\}$ in $n$ pairs, and thereby an element of $V_{2 n, n}$ is associated. The pairing between the elements associated with the two halves of $L$ is $P(L)$. 


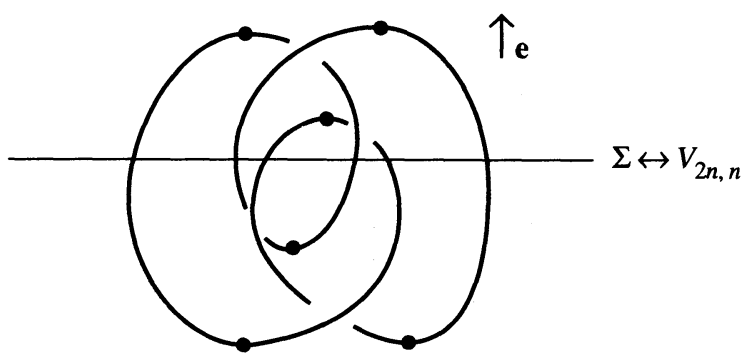

FIGURE 13

\section{Further remarks}

For any Lie algebra $\mathfrak{g}$ and representation $V$, an invariant of links can be constructed (see [9], [10] and [11]). When $\mathfrak{g}=\mathfrak{s l}_{2}$ and the spin-1/2 representation is used, the invariant obtained is the one-variable Jones polynomial $V_{L}(q)$. For $\mathfrak{g}=\mathfrak{s l}_{m}$ and the vector ( $m$-dimensional) representation, one obtains $X_{L}\left(q, q^{m-1}\right)$. The methods of [5] and $\S 3$ lead to the special case of the representation of the category of tangles in [9] and [11] appearing from $\mathfrak{s l}_{2}$ as above. However the methods of [5] can be extended to produce representations of $H_{n}(q)$ associated with multi-row Young diagrams, as well as to more complex representations of $B_{n}$ which do not factor through a quadratic algebra (see [6], [7]). This allows an extension of the methods of this paper, and gives rise to a topological construction of the two-variable Jones polynomial $X_{L}\left(q, q^{m-1}\right)$. For more details, see [7].

The case of $V_{L}(q)=X_{L}(q, q)$ is particularly simple because of the way in which the local systems introduced in the constructions of $\S 3$ involve twistings which do not distinguish between points in an object in $\mathbb{C T}$ with different orientations. This is due to the self-duality of $\mathfrak{s l}_{2}$, and consequently the corresponding construction for the two-variable Jones polynomial $X_{L}$ is more complex. It should be noted that this relates to the insensitivity of $V_{L}$ to a total reversal of orientation of $L$, that is, a change of $S^{1}$-orientation, as opposed to $S^{3}$-orientation to which $V_{L}$ is particularly sensitive. The Alexander polynomial $\Delta_{L}(q)=X_{L}\left(q, q^{-1}\right)$, on the other hand, is not sensitive to $S^{3}$-orientation, and is closely related (see for example [5]) to the local system on $\mathbb{C} \backslash \mathbf{w}$ in which the twists around $w_{i}$ are $q$ or $q^{-1}$ according to the orientation of the $i$ th strand. 


\section{References}

[1] V. G. Drinfel'd, Quantum groups, Proc. Internat. Congr. Math. (Berkeley, 1986), Amer. Math. Soc., Providence, RI, 1986, 798-820.

[2] M. Jimbo, $A$ q-analogue of $U(\mathfrak{g l}(N+1))$, Hecke algebras and the Yang-Baxter equations, Lett. Math. Phys. 11 (1986) 247-252.

[3] V. F. R. Jones, A polynomial invariant for knots via von Neumann algebras, Bull. Amer. Math. Soc. (N.S.) 12 (1985) 103-111.

[4] __ Hecke algebra representations of braid groups and link polynomials, Ann. Math. (2) 126 (1987) 335-388.

[5] R. J. Lawrence, Homology representations of braid groups, D. Phil. Thesis, Oxford (June 1989); Homological representations of the Hecke algebra, Comm. Math. Phys. 135 (1990) 141-191.

[6] __ The homological approach applied to higher representations, Harvard Univ. preprint (1990).

[7] __, Braid group representations associated with $\mathfrak{s l}_{m}$, Harvard Univ. preprint (1990).

[8] N. Yu. Reshetikhin, Quantised universal enveloping algebras, the Yang-Baxter equation, and invariants of links. I, II, LOMI Preprint E-4-87 I, II (1988).

[9] N. Yu. Reshetikhin and V. G. Turaev, Invariants of 3-manifolds via link polynomials and quantum groups, Invent. Math. 103 (1990) 547-597.

[10] V. G. Turaev, The Yang-Baxter equation and invariants of links, Invent. Math. 92 (1988) 527-553.

[11] _ _ The Conway and Kauffman modules of the solid torus with an appendix on the operator invariants of tangles, LOMI Preprint Leningrad E-6-88.

[12] H. Wenzl, Hecke algebras of type $A_{n}$ and subfactors, Invent. Math. 92 (1988) 349-383.

[13] E. Witten, Some geometrical applications of quantum field theory, IXth Internat. Congr. on Mathematical Physics, Springer, Berlin, 1989, 77-116.

[14] D. N. Yetter, Markov algebras, Contemp. Math., No. 78, Amer. Math. Soc., Providence, RI, 1988, 705-730.

HARVARD UNIVERSITY 